\title{
Spatial Multiplex in Downlink Multiuser Multiple-Antenna Wireless Environments
}

\author{
Wei Yu \\ Electrical and Computer Engineering Department, University of Toronto \\ 10 King's College Road, Toronto, Ontario M5S 3G4, Canada \\ email: weiyu@comm.utoronto.ca
}

\begin{abstract}
This paper studies the optimal spatial multiplex scheme in a downlink multiple-antenna environment with perfect transmitter and receiver channel knowledge. Using recent results on the sum capacity of the Gaussian vector broadcast channel, the optimal number of precoded data streams in a downlink channel is characterized. The main result is the following: the sum-capacity achieving transmission strategy in a random downlink channel with $n$ transmit antennas at the base-station and $K$ receivers each equipped with $m$ antennas involves between $n$ to $\frac{1}{2} n(n+1)$ data streams in total, with each user receiving between $m$ to $\frac{1}{2} m(m+1)$ data streams. This gives a dimension counting interpretation for multiuser diversity. In particular, it shows that the throughput maximizing transmission strategy in a downlink channel with $n$ transmit antennas should involve between $n$ to $\frac{1}{2} n(n+1)$ active users at any time.
\end{abstract}

\section{INTRODUCTION}

Multiple antennas provide an effective way to enhance the performance of a wireless system. Multiple antennas create extra dimensions in the spatial domain, and these dimensions can be used in many different ways. Multiple spatial dimensions can carry duplicate copies of the same information, thus increasing the reliability of information transmission, or the extra spatial dimensions can carry independent information in multiple data streams, thus increasing the data rate. The former technique is known as spatial diversity, the latter spatial multiplex. In general, there is a tradeoff between the two [1] [2].

The concept of diversity and spatial multiplex is also applicable to multiuser systems. Consider a cellular system with one base-station and many remote terminals geographically scattered in a single cell. Diversity in this multiuser system may be understood as follows: since not all remote terminals are likely to experience deep fades at the same time, the total throughput of the multiuser system is resilient to channel fading. Thus, diversity occurs not only across the antennas within each user, but also across the different users. This type of diversity is referred to as multiuser diversity [3]. It should be noted that multiuser diversity differs from single-user spatial diversity in one crucial aspect. In a single-user system, spatial diversity refers to the ability for the multiple antennas to transmit or receive the same information across several paths, while in a multiuser system, independent

This work was supported by NSERC and by the Canada Research Chair program. information is transmitted and received by different users. Thus, a multiuser system necessarily operates in a spatial multiplex mode whenever more than one users are active at the same time. This gives rise to the following question which was first raised in [4]. In a downlink spatial multiplex system with multiple transmit antennas, how many users should the base station accommodate at any given time? This question is important particularly for the design of the medium-access-control (MAC) protocols in a wireless network. The purpose of this paper is to develop a rigorous theory to tackle this question.

The concept of multiuser diversity appeared in Tse's work [3] on the downlink scheduling of Qualcomm HDR (High Data Rate) system. Information theoretical analysis shows that the sum-rate maximizing transmission strategy in a downlink channel with a single antenna at the base-station is the one where only a single user is active at the same time. Thus, [3] concentrated on how to best select the transmitting user. The goal of this paper is to generalize this idea to multi-antenna systems. For a downlink channel with $n$ transmit antennas, it is reasonable to postulate that the optimal transmission strategy should involve only $n$ users receiving at the same time. However, this is not true. In [4], Caire and Shamai exhibited a counter-example in which the sum-capacity achieving strategy involves more users than transmit antennas. The main result of this paper is to show that the maximum number of users that can be accommodated is bounded above. In particular, the rate sum maximizing transmission strategy in an $n$-antenna downlink channel can involve up to $\frac{1}{2} n(n+1)$ active users at the same time.

In the uplink direction, Knopp and Humblet [5] showed that in a single-antenna multiuser fading channel, the optimal transmission strategy is a TDMA-like scheme with a single user transmitting at a time. This result has since been generalized to the multi-antenna case in [6] [7], where it is shown that up to $\frac{1}{2} n(n+1)$ multiple users should transmit simultaneously if the base-station is equipped with $n$ antennas. The main result of this paper is a counter-part in the downlink channel. It relies on recent information theoretical results on the sum capacity of Gaussian vector broadcast channels [8] [9] [10] [11], and on a duality of the uplink and downlink channels [12] [11]. The main result also provides insights on the interaction between spatial diversity and multiuser diversity. 


\section{Multi-Antenna Wireless Environment}

Consider a cellular wireless environment, with a basestation equipped with $n$ antennas and $K$ remote terminals each equipped with $m$ antennas. The base-station-toremote-terminal transmission is modeled as follows:

$$
\mathbf{y}_{k}(i)=H_{k}(i) \mathbf{x}(i)+\mathbf{n}_{k}(i), \quad k=1 \cdots K,
$$

where $i$ denotes the time index, $\mathbf{x}(i)$ is a $n$-dimensional vector representing the transmitted signal from the basestation, $\mathbf{y}_{k}(i)$ is a $m$-dimensional vector representing the received signal for $k$ th user, and $\mathbf{n}_{k}(i)$ is the i.i.d. Gaussian noise. $H_{k}(i)$ is an $n \times m$ matrix, and it denotes the channel for user $k$ at time instant $i$. Note that the base-station jointly encodes independent information for all users, as all transmitting antennas are co-located. Remote terminals are scattered geographically, so they cannot coordinate.

Intuitively, as each user experiences a different channel, it is sensible to select only a subset of good channels at each time instant. The objective of this paper is to give a rigorous information theoretical analysis on how many users should be active at any given time. The main result is the following:

Theorem 1: Consider a Gaussian broadcast fading channel with $n$ transmit antennas, $K$ remote terminals each equipped with $m$ receive antennas and with i.i.d. Gaussian fading coefficients. With probability one, the sum-capacity achieving transmission scheme involves at most $\frac{1}{2} n(n+1)$ data streams in total, with each remote terminals using at most $\frac{1}{2} m(m+1)$ data streams. In particular, at most $\frac{1}{2} n(n+1)$ remote terminals can be active at any given time.

Two simplifying assumptions are made in deriving the above result, First, perfect and instantaneous channel state information is assumed to be available at both the base-station and remote terminals. Second, the sum rate is used as the criterion in the analysis. Although a real system design has to take into account channel estimation error and the performance tradeoff among the users, these two assumptions provide an amenable framework under which theoretical analysis can be made.

\section{Broadcast Channel Sum Capacity}

Consider the downlink channel illustrated in Fig. 1:

$$
\mathbf{y}_{\mathbf{k}}=H_{k} \mathbf{x}+\mathbf{n}_{\mathbf{k}}, \quad k=1 \cdots K
$$

where $\mathbf{x}, \mathbf{y}_{\mathbf{k}}$ and $\mathbf{n}_{\mathbf{k}}$ are vector valued, and $H_{1}, \cdots H_{K}$ are matrices. The transmitted signal is subject to a power constraint $\mathbf{E}\left[\mathbf{x}^{T} \mathbf{x}\right] \leq P$. Independent information is to be transmitted to each user. The capacity region refers to the set of rate tuples $\left(R_{1}, \cdots, R_{K}\right)$ simultaneously achievable by users 1 to $K$ with arbitrarily small probabilities of error. This paper concentrates on the rate sum $R_{1}+\cdots+R_{K}$.

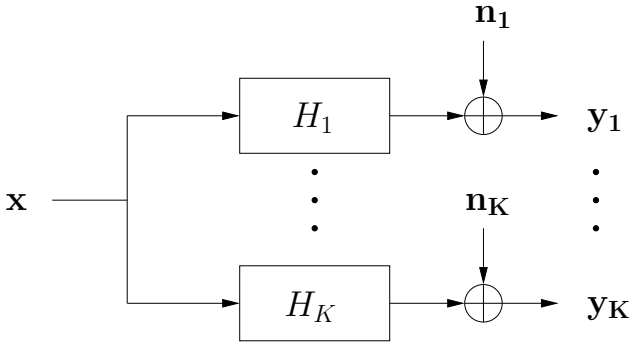

Fig. 1. The vector broadcast channel

The broadcast channel capacity is a long-standing open problem in network information theory. The capacity region for a broadcast channel is known only in several special cases (such as the degraded broadcast channel.) The Gaussian vector broadcast channel considered in this paper where the transmitter and receivers are equipped with multiple antennas is not necessarily degraded, and the capacity region is still not known completely. However, several recent work [8] [9] [10] [11] has successfully solved the sum capacity case.

There are two simultaneous and independent solutions to the sum capacity problem. A key ingredient in both solutions is a connection between the broadcast channel and channels with side information, first published in [8]. In a classic result known as "writing on dirty paper", Costa [13] showed that if a Gaussian channel is corrupted by an interference signal $\mathbf{s}$ that is known non-causally to the transmitter but not to the receiver, i.e.

$$
\mathbf{y}=\mathbf{x}+\mathbf{s}+\mathbf{n}
$$

the capacity of the channel is the same as if $\mathbf{s}$ does not exist. Thus, in a broadcast channel, if we let $\mathbf{x}=$ $\mathbf{x}_{1}+\cdots+\mathbf{x}_{K}$, where $\mathbf{x}_{k}$ is intended for the $k$ th user, the capacity of the channel from $\mathbf{x}_{k}$ to $\mathbf{y}_{k}$ is as large as if multiuser interference $\mathbf{x}_{1}, \cdots, \mathbf{x}_{k-1}$ can all be pre-subtracted. This precoding strategy turns out to be optimal for sumcapacity in a Gaussian broadcast channel. This is proved for the 2-antenna case by Caire and Shamai [8], and has since been generalized by several authors [9] [10] [11] using two different approaches. In the rest of this section, the decision-feedback approach [9] is reviewed first. Then, a new derivation for the duality approach (which was originally given in [10] and [11]) is presented.

\section{A. Decision-Feedback Equalization Approach}

The approach in [9] is based on the observation that multiuser interference pre-subtraction at the transmitter is similar to a decision-feedback equalizer at the receiver. The interference cancellation operation can in effect be "moved" to the transmitter. However, although the decision-feedback structure achieves the capacity of a Gaussian vector channel, it also requires coordination among the receivers because the DFE structure has a 
feedforward matrix that operates on all of $\mathbf{y}_{1}, \cdots, \mathbf{y}_{K}$. Clearly, such coordination is not possible in a broadcast channel. However, precisely because $\mathbf{y}_{1}, \cdots \mathbf{y}_{K}$ cannot coordinate, they are also ignorant of the noise correlation between $\mathbf{n}_{1}, \cdots, \mathbf{n}_{K}$. Thus, the sum capacity of the broadcast channel must be bounded by the cooperative capacity with the worst possible noise correlation, i.e.

$$
C_{\text {sum }} \leq \min _{S_{n n}} I\left(\mathbf{X} ; \mathbf{Y}_{1}, \cdots \mathbf{Y}_{K}\right)
$$

where $S_{n n}$ is the covariance matrix for $\mathbf{n}=\left[\mathbf{n}_{1}^{T} \cdots \mathbf{n}_{\mathbf{K}}^{T}\right]^{T}$, and the minimization is over all $S_{n n}$ whose $k$ th block diagonal term is the covariance matrix of $\mathbf{n}_{k}$. This outer bound is due to Sato [14]. Sato's outer bound can be evaluated explicitly by writing down the Karush-KuhnTucker (KKT) condition for the minimization problem:

$$
S_{n n}^{-1}-\left(H S_{x x} H^{T}+S_{n n}\right)^{-1}=\left[\begin{array}{ccc}
\Psi_{1} & & 0 \\
& \ddots & \\
0 & & \Psi_{K}
\end{array}\right],
$$

where $\Psi_{k}$ are the Lagrangian dual variables corresponding to the diagonal constraints. Interestingly and surprisingly, $S_{n n}^{-1}-\left(H S_{x x} H^{T}+S_{n n}\right)^{-1}$ also corresponds to the feedforward matrix of the decision-feedback equalizer! So, if the noise covariance happens to be the worst-case noise, the feedforward matrix of the decision-feedback equalizer would be diagonal. Thus, after moving the feedback operation to the transmitter, the entire equalizer de-couples into independent receivers for each user, and no coordination is needed whatsoever. Consequently, Sato's outer bound is achievable. Now, this achievable rate may be further maximized over all $S_{x x}$ subject to the power constraint. Therefore, the sum capacity of a Gaussian vector broadcast channel is:

$$
\begin{array}{cl}
\max _{S_{x x}} \min _{S_{n n}} & \frac{1}{2} \log \frac{\left|H S_{x x} H^{T}+S_{n n}\right|}{\left|S_{n n}\right|} \\
\text { subject to } & S_{n n}^{(i)}=I, \quad i=1, \cdots, K, \\
& \operatorname{trace}\left(S_{x x}\right) \leq P, \\
& S_{x x}, S_{n n} \geq 0,
\end{array}
$$

where $H=\left[H_{1}^{T} H_{2}^{T} \cdots H_{K}^{T}\right]^{T}$ and $S_{n n}^{(i)}$ refers to the $i$ th block-diagonal term of $S_{n n}$.

\section{B. Duality Approach}

The sum capacity of a Gaussian vector broadcast channel can be solved using a completely different method. In [12], it was observed that the achievable region of a broadcast channel using the precoding technique is exactly the same as the capacity region of a dual multiple access channel with the channel matrix transposed and a sum power constraint applied to all inputs. This uplink-downlink duality is closely related to convex duality. Based on the duality, [10] and [11] showed that the sum capacity of the broadcast channel is precisely the sum capacity of the dual multiple access channel. The proof also relies on Sato's outer bound. Duality is essential in our characterization of the structure of the optimal transmission strategy in the broadcast channel. In the rest of the section, we give a new derivation of duality that is different from that of [12] and [11].

To simplify matters, assume that $H$ is square and invertible. (The derivation can be generalized to arbitrary channels. See [15] and [16] for details.) The starting point of the new derivation is the minimax optimization problem (6). The objective function of the problem is concave in $S_{x x}$ and convex in $S_{n n}$, so its KKT conditions completely characterize the saddle points. The KKT conditions are:

$$
\begin{aligned}
& H^{T}\left(H S_{x x} H^{T}+S_{n n}\right)^{-1} H=\lambda I \\
& S_{n n}^{-1}-\left(H S_{x x} H^{T}+S_{n n}\right)^{-1}=\Psi
\end{aligned}
$$

where $\lambda$ is the dual variable associated with the power constraint and $\Psi=\operatorname{diag}\left(\Psi_{1}, \cdots, \Psi_{K}\right)$ is the dual variable associated with the diagonal constraint. Multiple (8) by $H^{T}$ on the left and $H$ on the right, substitute in (7), we obtain:

$$
H^{T} S_{n n}^{-1} H=H^{T} \Psi H+\lambda I .
$$

The above is equivalent to the following condition:

$$
H\left(H^{T} \Psi H+\lambda I\right)^{-1} H^{T}=S_{n n},
$$

where the transmit covariance matrix is a diagonal matrix $\Psi$, and the dual variable associated with the constraint has 1's on the diagonal (as the worst noise $S_{n n}$ has 1's on the diagonal.) This condition is precisely the KKT condition for a sum-power multiple access channel. After a proper scaling of the power constraint, $\Psi, \lambda$ and $S_{n n}$, it can be shown that the KKT condition of the following optimization problem is exactly (10):

$$
\begin{array}{cl}
\max _{D} & \frac{1}{2} \log \left|H^{T} D H+I\right| \\
\text { s.t. } & D \text { is diagonal } \\
& \operatorname{trace}(D) \leq P \\
& D \geq 0
\end{array}
$$

(where $D=\Psi / \lambda$.) Thus, the solution to the minimax problem (6) is just the solution to a maximization problem (11). This establishes the duality of a multiple access channel and a broadcast channel.

\section{Optimum Downlink Transmission Strategy}

The proof of the main theorem of the paper is now given. The strategy is to solve the sum power maximization problem (11) first, then show that the rank of the solution $D$ is at most $\frac{1}{2} n(n+1)$. Since $D$ is a scaled version of $\Psi$, and $\Psi$ is the diagonal matrix which corresponds 
to the feedforward section of the decision-feedback equalizer, we conclude that the decision-feedback equalizer for the broadcast channel can process at most $\frac{1}{2} n(n+1)$ data streams.

The key step in the argument is the characterization of the optimal $D$ in the sum-power multiple access channel capacity problem (11). The characterization is derived from its KKT condition. The KKT condition is:

$$
H_{k}\left(H^{T} D H+I\right)^{-1} H_{k}^{T}=\nu I+\Phi_{k}
$$

where $k=1 \cdots K$, and $\Phi_{k}$ is the slack variable for which

$$
\operatorname{trace}\left(\Phi_{k} D_{k}\right)=0
$$

Here, $D_{k}$ denotes the block diagonal entries of $D$ (i.e. $\left.D=\operatorname{diag}\left(D_{1}, \cdots, D_{K}\right)\right)$.

Our goal is to derive an upper bound on the number of non-zero diagonal entries of $D$. For simplicity, let's first consider the case where the receivers of the broadcast channel are each equipped with a single antenna only. Let the number of transmit antennas be $n$. Since $H_{k}$ is $1 \times n$, the matrix $\left(H^{T} D H+I\right)^{-1}$ is $n \times n$. But this matrix is common for each user, and it has at most $\frac{1}{2} n(n+1)$ degrees of freedom. This limits the maximum number of active users.

Lemma 1: Fix positive $\nu$, let $H_{1}, \cdots, H_{K}$ be $1 \times n$ random vectors with i.i.d. Gaussian entries. If $K>$ $\frac{1}{2} n(n+1)$, then with probability 1 , there does not exists a positive definite symmetric matrix $M$ such that $H_{k} M H_{k}^{T}=\nu, \forall k=1 \cdots K$.

This lemma is similar to an argument used in [6], where a detailed proof is given. Intuitively, a positive semidefinite matrix $M$ has $\frac{1}{2} n(n+1)$ degrees of freedom, but $K$ equations need to be satisfied simultaneously. This is possible only if $K \leq \frac{1}{2} n(n+1)$. The proof can be made rigorous by recognizing that $H_{k}$ are i.i.d. Gaussian, so with probability 1 , the $K$ equations are independent.

Now consider a fading environment with the basestation equipped with $n$ antennas. To maximize the sum capacity, water-filling must be done across the fading states. Such water-filling in time fixes the water level $\nu$. Now, apply Lemma 1 . Set $M=\left(H^{T} D H+I\right)^{-1}$. Lemma 1 implies that $\Phi_{k}=0$ for at most $\frac{1}{2} n(n+1)$ users. So, $D_{k}$ (and thus $\Psi_{k}$ ) is positive for at most $\frac{1}{2} n(n+1)$ users. Therefore, the rate-sum maximizing strategy must have at most $\frac{1}{2} n(n+1)$ active users at the same time.

Lemma 1 can be generalized to the case of multiple receive antennas in the same way as done in [6]. Parallel to the previous development, we ask whether there exists a positive semidefinite matrix $M$ that satisfies $H_{k} M H_{k}^{T}=$ $\nu I+\Phi_{k}$ for each $k=1, \cdots, K$. The idea is again to count the number of independent equations and the number of unknowns. The matrix equation has $\frac{1}{2} m(m+1)$ independent entries for each $k$, so there are in total $\frac{K}{2} m(m+1)$ independent equations. The number of unknown variables is counted as follows. The matrix $M$ introduces $\frac{1}{2} n(n+1)$ degrees of freedom. The number of unknowns introduced by the slack variable $\Phi_{k}$ depends on its rank. An $m \times m$ matrix with rank $r$ has $\frac{1}{2} m(m+1)-\frac{1}{2}(m-r)(m-r+1)$ unknowns. (To see this, recall that a positive semidefinite symmetric matrix can be represented by its Cholesky factorization as $L L^{*}$. If a $m \times n$ matrix is of rank $r$, its Cholesky factor is a $m \times r$ triangular matrix, with exactly $\frac{1}{2} m(m+1)-\frac{1}{2}(m-r)(m-r+1)$ independent entries. $)$ Now, the slack variables need to satisfy the complementary slackness condition (13). So, if the transmit signal $D_{k}$ is rank $r_{k}$, the rank of $\Phi_{k}$ is at most $m-r_{k}$. Therefore, each $\Phi_{k}$ introduces at most $\frac{1}{2} m(m+1)-\frac{1}{2} r_{k}\left(r_{k}+1\right)$ extra degrees of freedom. The total number of unknown variables is then $\frac{1}{2} n(n+1)$ from the matrix $M$ plus $\frac{1}{2} m(m+1)-\frac{1}{2} r_{k}\left(r_{k}+1\right)$ from each of $\Phi_{k}$. Now, since the channel realization $H_{k}$ is random, the equations are independent. Thus, for a solution to exist, there must be at least as many unknown variables as there are equations. So,

$$
\begin{aligned}
\frac{n(n+1)}{2}+K \frac{m(m+1)}{2} & -\sum_{k=1}^{K} \frac{r_{k}\left(r_{k}+1\right)}{2} \\
& \geq K \frac{m(m+1)}{2} .
\end{aligned}
$$

Then, it follows that $\sum_{k} r_{k}\left(r_{k}+1\right) \leq n(n+1)$. Since the rank $r_{k}$ is bounded by the number of receive antennas $m$, we conclude that in a random downlink environment with $n$ transmit antennas, $K$ remote terminals with $m$ receive antennas each, with probability one, the sum-rate optimal transmission scheme uses at most $\frac{1}{2} n(n+1)$ data streams in total, with each remote terminals using at most $\frac{1}{2} m(m+1)$ data streams. In particular, at most $\frac{1}{2} n(n+1)$ remote terminals can be active at the same time.

Finally, we remark that when there are sufficient amount of transmit power and sufficient number of receivers, uplink-downlink duality also implies that the minimum number of data streams is lower bounded by $n$. Thus, the optimal number of data streams is between $n$ and $\frac{1}{2} n(n+1)$.

\section{Simulation Results}

Theorem 1 is an upper bound on the total number of active receivers in a downlink multi-antenna broadcast channel. This upper bound is not necessarily tight. Fig. 2 and Fig. 3 show simulation results on a Rayleigh fading broadcast channel with multiple receivers each with a single-antenna and a base-station with varying number of transmit antennas. The optimization problem (11) is solved using a numerical approach called dual decomposition [17]. Fig. 2 plots the number of active users vs the number of transmit antennas over several realizations of the channel. It is seen that although the theoretical upper bound on the number of active users goes up quadratically with the number of antennas, the actual number of 


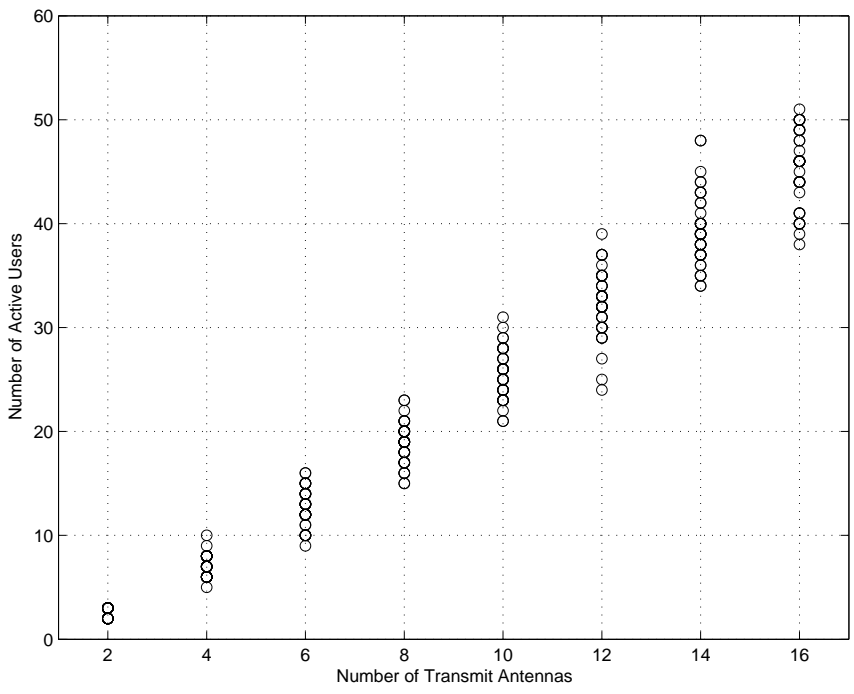

Fig. 2. The number of active users vs the number of transmit antennas over random realizations of the channel.

active users goes up roughly linearly. Fig. 3 shows a histogram of the number of active users over 100 random realizations of the channel for the cases of $2,4,8$ and 16 transmit antennas.

\section{Concluding Remarks}

When the base-station in a downlink cellular system is equipped with a single antenna, the sum rate maximizing transmission strategy should transmit to the user with the best channel. Thus, only one user is served at a time. The main point of this paper is to show that with multiple antennas at the base-station, the sum-rate optimal transmission strategy consists of multiple users being served simultaneously. Theorem 1 makes a precise dimension counting argument that ties in spatial diversity and multiuser diversity. In a random propagation environment, a downlink broadcast channel with $n$ transmit antennas has about $n$ to $\frac{1}{2} n(n+1)$ spatial dimensions. These dimensions are divided among the remote terminals. A remote terminal with $m$ receive antennas can utilize about $m$ to $\frac{1}{2} m(m+1)$ dimensions. The dimensions are additive, and the total number of dimensions is roughly between $n$ and $\frac{1}{2} n(n+1)$.

There is an interesting interplay between spatial diversity and multiuser diversity. Spatial dimensions are discrete resources. Multiple antennas at the base-station create dimensions which allow more than one remote terminals to transmit and receive simultaneously. Multiple antennas at the remote terminals, however, has the opposite effect. They improve the performance of each remote terminal at the expense of crowding out other users from transmitting and receiving at the same time.
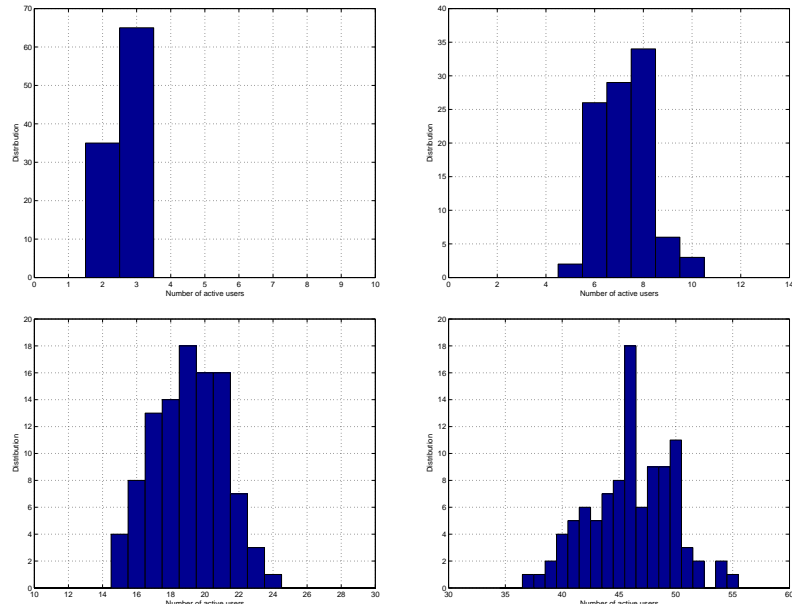

Fig. 3. Histograms of the number of active users over 100 random realizations of the channel. Top-left, top-right, bottom-left and bottom-right figures correspond to the cases of 2, 4, 8 and 16 transmit antennas respectively.

\section{REFERENCES}

[1] L. Zheng and D. Tse, "Diversity and multiplexing: A fundamental tradeoff in multiple antenna channels," IEEE Trans. Info. Theory, vol. 49, pp. 1073-96, May 2003.

[2] R. Heath Jr and A. Paulraj, "Switching between multiplexing and diversity based on constellation distance," in Allerton Conf. Comm. Cont. Comp., 2000.

[3] D. Tse, "Downlink scheduling for Qualcomm HDR system," 1999.

[4] G. Caire and S. Shamai, "On the multiple antenna broadcast channel," in Asilomar Conf. Signal System Computers, 2001.

[5] R. Knopp and P. A. Humblet, "Information capacity and power control in single-cell multi-user communications," in IEEE Inter. Conf. Comm. (ICC), 1995, pp. 331-335.

[6] W. Yu, W. Rhee, and J. M. Cioffi, "Optimal power control in multiple access fading channels with multiple antennas," in IEEE Inter. Conf. Comm. (ICC), 2001.

[7] W. Rhee, W. Yu, and J. M. Cioffi, "The optimality of beamforming in uplink multiuser wireless systems," to appear in IEEE Trans. Wireless, 2001.

[8] G. Caire and S. Shamai, "On the achievable throughput of a multi-antenna Gaussain broadcast channel," IEEE Trans. Info. Theory, July 2003.

[9] W. Yu and J. M. Cioff, "Sum capacity of Gaussian vector broadcast channels," to appear in IEEE Trans. Info. Theory.

[10] S. Vishwanath, N. Jindal, and A. Goldsmith, "Duality, achievable rates and sum-rate capacity of Gaussian MIMO broadcast channels," to appear in IEEE Trans. Info. Theory, 2003.

[11] P. Viswanath and D. Tse, "Sum capacity of the multiple antenna Gaussian broadcast channel and uplink-downlink duality," IEEE Trans. Info. Theory, August 2003.

[12] N. Jindal, S. Vishwanath, and A. Goldsmith, "On the duality of Gaussian multiple-access and broadcast channels," to appear in IEEE Trans. Info. Theory, 2003.

[13] M. Costa, "Writing on dirty paper," IEEE Trans. Info. Theory, vol. 29, no. 3, pp. 439-441, May 1983.

[14] H. Sato, "An outer bound on the capacity region of broadcast channels," IEEE Trans. Info. Theory, May 1978.

[15] W. Yu, "The structure of the worst-noise in Gaussian vector broadcast channels," in DIMACS Workshop on Network Info. Theory, 2003.

[16] W. Yu, "Uplink-downlink duality via minimax duality," in Canadian Workshop on Info. Theory, 2003.

[17] W. Yu, "A dual decomposition approach to the sum power multiple access channel sum capacity problem," in Conf. Info. Sci. Sys. (CISS), 2003. 